\title{
CDISC SDTM Coronary Thrombus TIMI Grade Responses Terminology
}

National Cancer Institute

\section{Source}

National Cancer Institute. CDISC SDTM Coronary Thrombus TIMI Grade Responses

Terminology. NCI Thesaurus. Code C119017.

Terminology associated with the coronary thrombus TIMI grade responses codelist of the Clinical Data Interchange Standards Consortium (CDISC) Study Data Tabulation Model (SDTM). 